\title{
The role of the prefrontal cortex in dynamic filtering
}

\author{
ARTHUR P. SHIMAMURA \\ University of California, Berkeley, California
}

\begin{abstract}
Functional neuroimaging and neuropsychological methods have broadened our understanding of the human prefrontal cortex. Converging evidence suggests that this brain region contributes to executive control of information processing. Both cognitive and neural-based models have attempted to delineate the manner in which the prefrontal cortex mediates executive control. An analysis of these findings and models suggests four prominent aspects of executive control-selecting, maintaining, updating, and rerouting information processing. These four aspects are couched in terms of dymamic filtering theory, which proposes that the prefrontal cortex acts as a selective gating or filtering mechanism that controls information processing.
\end{abstract}

Advances in cognitive neuroscience have begun to clarify the role of the prefrontal cortex in human behavior. Early theorists viewed this brain region as the seat of human intellect and abstract reasoning (Goldstein, 1936; Halstead, 1947). This viewpoint was influenced by the observation that through evolution the size of the prefrontal cortex increased out of proportion to other brain regions. Indeed, in primates, the size of the prefrontal cortex ranges from $11.5 \%$ of the neocortex in macaque monkeys to $29 \%$ of the neocortex in humans (Fuster, 1989). Yet its role in general intellectual reasoning became suspect, because damage to the prefrontal cortex did not grossly affect measures of IQ (Hebb, 1945; Janowsky, Shimamura, Kritchevsky, \& Squire, 1989; but see Duncan, Emslie, Williams, \& Johnson, 1996).

Other factors kept prefrontal function in the purview of a "riddle." First, this brain region could not be tied specifically to either sensory or motor functions (Teuber, 1964). Second, it could not be tied specifically to any particular cognitive function (see Stuss \& Benson, 1986). Indeed, findings from extensive neuropsychological investigations indicate that damage to the prefrontal cortex produces a plethora of mental dysfunctions, including disorders of attention, memory, language, problem solving, affect, and response selection (Luria, 1966; Milner, Petrides, \& Smith, 1985; Shimamura, 1994; Stuss, Eskes, \& Foster, 1994).

It is not too surprising that a variety of psychological dysfunctions have been associated with prefrontal damage, since this brain region comprises such a large proportion of the neocortex. In fact, it is reasonable to suggest that different regions of the prefrontal cortex contribute to mental activity in different ways. Anatomically, the pre-

This research was supported by NIH Grants MH48757 and NS17778 to A. P. Shimamura. I thank Mark D'Esposito, Rich Ivry, and Robert Knight for useful discussions of the issues presented. Correspondence concerning this article should be addressed to A. P. Shimamura, Department of Psychology (1650), University of California, Berkeley, CA 94720 (e-mail: aps@socrates.berkeley.edu). frontal cortex is defined as the area in the frontal lobes that receives projections from the mediodorsal nucleus of the thalamus. The human prefrontal cortex can be divided into three primary regions - the dorsolateral region, orbitofrontal region, and the frontal eye fields. The dorsolateral region, which is the largest part, comprises BA9, BA10, BA44, BA45, and BA46 (BA, Brodmann areas). The orbitofrontal region includes the anterior ventral and medial surface of the frontal lobes and comprises BA1 1, BA12, and BA47. This region is also described as the ventromedial prefrontal cortex (Damasio, 1995). The frontal eye field is generally defined as BA8. These three regions in the prefrontal receive projections from different regions within the mediodorsal nucleus of the thalamus (Fuster, 1989). Other cytoarchitectonic maps have been developed for the macaque monkey (e.g., Bonin \& Bailey, 1947; Walker, 1940), and comparative analyses of human and monkey prefrontal regions have been accomplished. Detailed description of anatomical findings and comparative analyses are beyond the scope of this review (for further information, see Fuster, 1989; Pandya \& Yeterian, 1998; Petrides \& Pandya, 1994).

During the 1960s and 1970s, Mishkin and colleagues (Mishkin, 1964; Mishkin \& Manning, 1978) refined the role of the prefrontal function by demonstrating that the dorsal and ventral regions serve different cognitive functions. Lesion studies using primate models defined a dorsal region that comprises the area surrounding and above the sulcus principalis (WA46 and WA9, Walker areas). A ventral region was initially defined as the region below the sulcus principalis-specifically, the inferior convexity (WA45 and WA12) and the ventromedial surface (WA13). Later, more restricted lesion studies defined the ventral region as the inferior convexity alone (excluding WA13). In these studies, lesions in the dorsal region disrupted spatial memory, which was measured by the delayedresponse task (Jacobson, 1936). In this task, food is placed in one of two locations, and the animal must remember the correct location after delays of several seconds. This task has been studied elegantly by Fuster, Goldman-Rakic, 
Miller, and others (see Fuster, 1989; Goldman-Rakic, 1998; Miller, Erickson, \& Desimone, 1996). Lesions in the ventral region produce an abnormal perseveration of central sets (Mishkin, 1964). A hallmark feature of this deficit is an inability to shift sets in object reversal and go/no-go tasks. In such tasks, the animal must inhibit an incorrect but dominant response tendency. In human studies, set-shifting tasks have been used to assess the ability to disengage from one task and perform another.

In the past decade, research on human prefrontal function has been one of the most active areas in cognitive neuroscience (for review, see Miller \& Cummings, 1999; Roberts, Robbins, \& Weiskrantz, 1998). Most of these investigations have centered on the role of the dorsolateral prefrontal cortex in executive control - that is, the selection, monitoring, and control of cognitive processes. Neuropsychological studies of patients with frontal lobe lesions and functional neuroimaging studies of neurologically intact individuals suggest that the dorsolateral region is involved in tasks that require various control processes, such as stimulus selection, working memory, memory retrieval, and set shifting. Executive control is presumed to enable top-down "supervision" of cognitive processing at various stages, such as perceptual analysis, short-term memory, and response selection.

The most influential characterization of executive control is Baddeley's model of working memory (Baddeley, 1986). Working memory refers to the processes and representations required for the temporary storage of information. This concept is related to short-term memory, which, in this paper, is a term used to describe specifically the temporary activation of memory representations (i.e., active representations, but not executive processes). According to Baddeley, working memory is characterized by two storage buffers and a control mechanism. One buffer, the phonological loop, holds phonological information, whereas the other buffer, the visuospatial sketchpad, holds visuospatial information. These two buffers are controlled by the central executive. The central executive selects and maintains information in the buffers. Thus, control is based on what is stored temporarily in the buffers, which is not unlike the way a digital computer uses temporary RAM buffers to store information. Baddeley suggests that patients with frontal lobe lesions exhibit a dysexecutive syndrome, in which there is a failure to control the selection of information in temporary storage.

\section{ASPECTS OF EXECUTIVE CONTROL}

In this paper, I describe four aspects of executive control-selecting, maintaining, updating, and rerouting information processing. Selecting refers to the ability to focus attention on aspects of information processing, such as attending to stimulus events or memory representations. Maintaining refers to the ability to keep active information in short-term memory after it has been selected. Updating refers to the modulation and reorganization of information in short-term memory. Rerouting refers to the ability to switch from one cognitive process or response set to another. It is proposed that these four aspects of executive control are arranged by level of complexity, from the most rudimentary aspect of control (i.e., selecting) to the most demanding aspect (i.e., rerouting). Moreover, it is suggested that the neural basis of executive control can be explained by a dynamic filtering theory, which proposes that the prefrontal cortex filters or gates neural activity in the posterior cortical regions.

\section{Selecting Activity and Focusing Attention}

Selective attention refers to the focussing of attention to perceptual features or to information in memory. A classic paradigm that assesses selective attention is the Stroop color-word test (see MacLeod, 1991; Stroop, 1935). In this and similar "Stroop-like" tests, subjects attend to one stimulus feature (e.g., the color of the words) and ignore another (e.g., the words themselves). Perret (1974) assessed patients with frontal lobe lesions on the Stroop color-word test and found increased interference effects in these patients. That is, the patients exhibited particularly long response latencies when asked to name the color of the stimuli when the stimuli were themselves incongruous names of colors. Although this finding has stood as a cornerstone for many years, other studies have not always replicated it (Baldo \& Shimamura, 1995; Dunbar \& Sussman, 1995; Shallice, 1982; Stuss et al., 1994). Problems in scaling differential baseline latencies between controls and patients and heterogeneity of patient groups have prevented conclusive results of either normal or disproportionate interference on Stroop tests.

The flanker task, which is related to the Stroop test, has shown some sensitivity for a selection deficit in patients with frontal lobe lesions. In this task, subjects respond to a central target stimulus (e.g., a color patch) in the presence of an adjacent "flanker" stimulus (e.g., another color patch). The flanker stimulus may be congruent or incongruent with the target stimulus. For control subjects, response latencies are faster when the flanker is congruent with the target than when it is incongruent. Rafal, Gershberg, Egly, and Ivry (1996) assessed patients with unilateral prefrontal lesions and observed the flanker effect when the flanker was presented in the ispilesional visual field (i.e., to the nonlesioned hemisphere), but there was no effect when the flanker was presented in the contralesional field (i.e., to the lesioned hemisphere). This finding suggests a narrowing of the attentional window in patients with frontal lobe lesions.

Neuroimaging studies of the Stroop and flanker tasks have more reliably activated anterior cingulate regions rather than prefrontal regions (Bench, Frith, Grasby, \& Friston, 1993; Botvinick, Nystrom, Fissell, \& Carter, 1999; Peterson, Bradley, Skudlarski, Gratenby, \& Zhang, 1999). However, regional activation in polar and medial frontal 
areas have also been activated. There is some controversy concerning the degree to which these tasks involve the selection of perceptual features or response modes. It may be that perceptual or response filtering is less a purview of the prefrontal cortex and is more associated with the anterior cingulate cortex. Perhaps, selection of information in working memory may be more rooted in prefrontal processing. In summary, neuropsychological and neuroimaging studies suggest evidence for the role of the anterior cingulate cortex in filtering perceptual or response modes, and there is some evidence for a contributory role of the prefrontal cortex in supporting these functions.

\section{Maintaining Activity in Short-Term Memory}

There is a strong interrelationship between selective attention and short-term memory. It could be argued that short-term memory is the informational content that is activated via selective attention. In tasks involving shortterm memory, information is not only selected, but also kept active for a brief period (i.e., seconds) until a response is required. Immediate span tasks, such as the digit span test, are benchmark measures of short-term memory. In such tasks, subjects listen to a string of $4-8$ digits and must report the digits immediately after presentation. In animal models, the delayed response task has served as a benchmark test of this process. This notion of working memory-that is, the ability to maintain neural activity after a stimulus has been presented-has been used to describe prefrontal function in primate models (see Fuster, 1989; Goldman-Rakic, 1998).

Patients with frontal lobe lesions are impaired on immediate span tasks. Janowsky et al. (1989) assessed a group of patients with unilateral or bilateral frontal lobe lesions and observed impairment on standard tests of digit span. Ptito, Crane, Leonard, Amsel, and Caramanos (1995) assessed patients with frontal lobe lesions on a spatial memory task, similar to one used in primate studies (Funahashi, Bruce, \& Goldman-Rakic, 1993). On each trial, subjects were presented a stimulus in the periphery, and after a delay (up to $30 \mathrm{sec}$ ), they were asked to indicate the location of the stimulus. The patients with frontal lobe lesions were less accurate than the control subjects on this task. Baldo and Shimamura (2000) observed impaired performance in patients with frontal lobe lesions on both spatial and color short-term memory tasks. In that study, performance at the zero-delay condition was often impaired in patients with frontal lobe lesions, suggesting a deficit in the initial encoding or selection of the target stimulus. Chao and Knight (1996) observed impaired performance in patients with frontal lobe lesions on an auditory short-term memory task. In these span studies, deficits were often exacerbated by the presentation of distracting stimuli during the delay period.

Some reports have failed to observe deficits on tasks involving immediate span. Recently, however, D'Esposito and Postle (1999) performed a meta-analysis of reported findings of span task performance in patients with frontal lobe lesions. Across the studies, a total of $116 \mathrm{pa}-$ tients were assessed on span tasks. D'Esposito and Postle (1999) found significant deficits in immediate span tasks in frontal patients compared with control subjects. Failure to observe deficits in some individual studies were likely a result of test insensitivity due to a limited range in test scores (all subjects tend to perform between 4 and 8 items correct) and to the limited number of patients assessed in a study.

Neuroimaging studies have affirmed the role of the prefrontal cortex in maintaining activity in short-term memory. Both PET and fMRI findings have identified prefrontal regions involved in the active maintenance of spatial, object, and verbal information (Awh, Jonides, Smith, Schumacher, \& Koeppe, 1996; Jonides et al., 1993; McCarthy, Blamire, Rothman, Gruetter, \& Shulman, 1993; Smith et al., 1995). Interestingly, there appears to be some regional specificity in terms of the informational content that is maintained. Smith et al. found increased left-hemisphere activation for object short-term memory, but increased right-hemisphere activation for spatial short-term memory. Moreover, for spatial shortterm memory, activity in the posterior parietal cortex (BA40) was apparent, but for object short-term memory, posterior activity occurred in the inferior temporal cortex (BA 37). These findings suggest a neural circuitry involving the participation of prefrontal regions with posterior cortical regions. The findings fit well with primate models that suggest separate pathways for spatial and object information processing (Ungerleider \& Mishkin, 1982; Wilson, O'Scalaidhe, \& Goldman-Rakic, 1993), though it is important to note that both separation and integration of these cortical streams may be critical for executive control (see Rainer, Asaad, \& Miller, 1998; Rao, Rainer, \& Miller, 1997).

\section{Updating Activity: Manipulation of Information in Short-term Memory}

In many cognitive tasks, it is necessary to manipulate information in short-term memory. This process is critical for both short-term and long-term memory tasks. For example, the ability to recall the details of an event that has occurred weeks ago requires the manipulation and reorganization of activation in a way to gain access to other memories. During such strategic searches, certain retrieval cues, such as where the event took place or the people involved, may facilitate memory for other details of the event. Alternatively, some cues may fail to facilitate and must be rejected as retrieval aids. Thus, relevant information must be selected, maintained, and updated as processing continues. Updating refers to a specific aspect of manipulating, or modulating, activation in shortterm memory. Updating concerns the ability to alter activity levels among items in short-term memory. Thus, rather than merely maintaining activity, updating involves top-down reorganization of activation levels. 
In short-term memory tasks, updating has been assessed in the "self-ordered pointing" task (Petrides \& Milner, 1982). In this task, subjects are presented an array of stimuli (e.g., eight pictures of objects) and asked to point to one of the stimuli. On subsequent trials, the same stimuli are presented in different positions, and the subjects are asked to point to a stimulus, with the restriction that they must point to a different one on each trial. This task requires updating stimulus information. That is, after each trial, the contents in short-term memory must be reorganized to include another item. Patients with frontal lobe lesions and monkeys with mid-dorsal lesions (WA9 and WA46) exhibit impairment on this task (Petrides, Alivisatos, \& Evans, 1995; Petrides \& Milner, 1982). They fail to monitor (i.e., update) prior stimuli that they have previously responded to. In addition, in a PET study, dorsolateral prefrontal areas (BA9 and BA46) were activated when subjects performed this task (Petrides, Alivisatos, Evans, \& Meyer, 1993).

The $n$-back task is another short-term memory task involving memory updating. In this task, subjects are presented a series of stimuli, such as single letters. For each presentation, subjects are to respond as to whether or not the letter presented on the current trial is the same letter that has been presented $n$ trials ago. That is, for a 1-back task, subjects are to respond yes if the letter presented is the same one that was presented in the preceding trial. In a 2-back task, subjects are to respond yes if the letter presented is the same one that was presented two trials earlier. Dorsolateral prefrontal activation has been observed in this task in functional neuroimaging studies (Awh et al., 1996; Carlson, Martinkauppi, Raemae, \& Salli, 1998; Cohen, Perlstein, Braver, \& Nystrom, 1997). In addition to activating mid-dorsal areas, $n$-back tasks appear to activate other cortical and subcortical regions (e.g., the cerebellum) as task difficulty increases.

As mentioned earlier, updating is a critical executive function for many cognitive and memory tasks. Such tasks are generally disrupted by frontal lobe lesions. For example, in semantic retrieval tasks, such as those involving verbal fluency, subjects are given letter cues ("F" or "S") or category cues ("animals" or "fruits") and asked to retrieve as many words as possible within a 1 -min period (Benton \& deHamsher, 1976; Milner, 1964). As in the self-ordered pointing task, fluency tasks require subjects to monitor items previously responded to and to update this list after each response. Failure to monitor and update information leads to perseverations (i.e., repetitions of a prior response). Patients with frontal lobe lesions exhibit impairment on a variety of fluency tasks, including letter fluency, category fluency, and design fluency (Baldo \& Shimamura, 1998; Benton \& deHamsher, 1976; JonesGottman \& Milner, 1977; Milner, 1964; Owen, Downes, Sahakian, Polkey, \& Robbins, 1990). Moreover, in a PET study (Frith, Friston, Liddle, \& Frackowiak, 1991), verbal fluency performance has been associated with activation in the prefrontal cortex. Finally, in a unique study in which frontal lobe patients and functional neuroimaging were used, Thompson-Schill et al. (1998) found that the prefrontal cortex was critically involved in memory selection during a semantic retrieval task.

Updating is also critical for the learning and organization of recently acquired information. That is, efficient learning involves the reorganization of sensory information into meaningful or conceptual representations. This process requires the integration of new information with existing knowledge. As such, learning must involve updating and organization of both sensory information and existing knowledge. Patients with frontal lobe lesions lack efficient organizational strategies for learning (Eslinger \& Grattan, 1994; Gershberg \& Shimamura, 1995; Hirst \& Volpe, 1988; Moscovitch, 1994). Moreover, neuroimaging studies have demonstrated increased prefrontal activity during semantic encoding (Brewer, Zhao, Desmond, Glover, \& Gabrieli, 1998; Buckner, 1996; Demb et al., 1995; Gabrieli et al., 1996; Nyberg, Cabeza, \& Tulving, 1996; Wagner, Schacter, Rotte, \& Koutstaal, 1998).

Another aspect of memory that requires extensive updating is the phenomenon of source memory. In tasks involving this phenomenon, subjects are asked to recollect when or where they have obtained some information or taken part in some event, such as the last time they have attended a banquet (Johnson, Hashtroudi, \& Lindsay, 1993; Janowsky, Shimamura, \& Squire, 1989; Schacter, Harbluk, \& McLachlan, 1984). Remembering source information often involves updating in short-term memory, because direct retrieval of this information is unlikely and it is therefore necessary to develop search strategies to reconstruct time and place information. As in $n$-back and self-ordered pointing tasks, task-relevant information must be maintained, updated, and reorganized.

Problems in memory for temporal order and other source information are prominent features of frontal lobe damage (Janowsky, Shimamura, \& Squire, 1989; Shimamura, Janowsky, \& Squire, 1990). Also, neuroimaging studies have indicated prefrontal activation during source memory tasks (Nolde, Johnson, \& D'Esposito, 1998). In the past, a deficit in memory for temporal order has been viewed as a specific feature of frontal lobe pathology (Milner, 1971). Given the interpretation presented here, such deficits occur because the task requires extensive executive control (i.e., updating) of memory activations. Thus, memory for temporal order or source memory, per se, is not an inherent prefrontal function. Instead, patients with frontal lobe lesions exhibit problems in source recollection because such tasks depend critically on executive control of memory activations, in comparison with tasks that depend on familiarity-based memory (e.g., recognition or priming).

In summary, memory updating can be observed on various tasks, such as the self-ordered pointing, $n$-back, fluency, semantic retrieval, learning, and source memory tasks. Findings from both neuropsychological and neuroimaging studies suggest that the prefrontal cortex is prominently involved in both short-term and long-term 
memory tasks that involve the updating of memory activations. In these tasks, it is necessary to rearrange or modulate the level of activations in short-term memory.

\section{Rerouting Activity: Implementing Set Shifting}

Relative to selecting, maintaining, and updating, the ability to reroute information processing is the most demanding executive function. Rerouting involves a global shifting of activity, from stimulus selection to response selection. Set switching paradigms are benchmark tests of rerouting. In such tasks, subjects must alternate between two tasks across trials. Dunbar and Sussman (1995) assessed patients with frontal lobe lesions on a Strooplike picture-word paradigm. In the control condition, the subjects were asked always to name pictures of objects and to ignore simultaneously presented words. The patients with frontal lobe lesions (from closed head injuries) did not exhibit any greater interference on this Strooplike task in comparison with the control subjects. However, in a set-shifting variation of the task, the subjects were cued at the beginning of each trial as to whether they were to name the picture or read the word. The patients with frontal lobe lesions were particularly slow in the setshifting condition.

Set switching paradigms have become popular in studies of attention (Allport, Styles, \& Hsieh, 1994; Monsell, 1996). In these studies, subjects are presented stimuli that could be processed in different ways. For example, in a study by Duncan et al. (1996), the subjects monitored two streams of stimuli (random series of letters and digits). At the beginning of a stream, they were asked to report only the letters from one side. Later, a cue would indicate whether the subject was to switch and report the letters presented in the other stream. The patients with frontal lobe lesions exhibited difficulty in switching between left and right streams.

Findings from dual-task paradigms also suggest that the prefrontal cortex is prominently involved in rerouting. In these paradigms, subjects must monitor two tasks simultaneously. For example, in an fMRI study, D'Esposito, Detre, Alsop, and Shin (1995) asked subjects to perform a semantic judgment task (e.g., to monitor each time a type of vegetable was presented) and a visuospatial task (e.g., mental rotation) either separately or simultaneously. Significant prefrontal activation (BA9 and BA46) was observed when the subjects performed the two tasks simultaneously but not when they performed the two tasks separately. Dual-task conditions require extensive rerouting of information processing in order for subjects to perform efficiently on both tasks.

One of the benchmark neuropsychological tests of frontal lobe function, the Wisconsin Card Sorting Test (Milner, 1964), involves set shifting and rerouting of task demands. As in other tasks that involve rerouting, the stimuli used in the Wisconsin Card Sorting Test are multidimensional, and subjects must determine which stimulus dimension (color, shape, or number) is relevant. Pa- tients with frontal lobe lesions fail to appreciate shifts in the relevant dimension and tend to perseverate on previously rewarded dimensions. Similar tasks, such as the California sorting task (Delis, Squire, Bihrle, \& Massman, 1992) and concept identification tasks (Owen et al., 1993), also indicate problems in the ability to shift sets. In particular, patients with frontal lobe lesions have difficulty rerouting processing from a previously successful or dominant set. These findings echo previous studies by Mishkin (1964) on the perseveration of central sets in monkeys (see also Dias, Robbins, \& Roberts, 1996).

One aspect that makes rerouting a particularly complex executive function is the need to disengage from and shift to different processing modes. Thus, rerouting requires not only control of short-term memory, but also the control of processing sets and response programs. For example, to shift from word naming to color naming during a set-shifting version of the Stroop task, subjects must reroute processing and responses on the basis of task demands. Such internally mediated or volitional shifts in processing may be particularly demanding on executive control processes. Indeed, rerouting requires shifts in stimulus activations, stimulus processing, and response programming. As such, it represents one the most complex forms of executive control.

\section{DYNAMIC FILTERING THEORY A Theoretical Account of Prefrontal Function}

How can executive control be implemented in the brain? That is, what kind of neural architecture might enable top-down control of information processing? At the cognitive level, such terms as central executive and supervisor suggest a rather intelligent control mechanism that oversees and manages information processing. It is, of course, necessary to avoid an extreme homuncular view of a little person in the head monitoring and controlling cognition. Yet, it seems clear that top-down or volitional control of cognition exists. As a way to avoid an ill-defined homuncular view, it is important to describe succinctly the manner in which control is implemented. It is useful to note that even simple devices have the ability to monitor and control. Take for example, a thermostat that monitors the ambient temperature of a room and, depending upon its assessment, controls room temperature by engaging a heater or air conditioner. In this case, the "decision" to initiate control is contingent on the state of the environment.

By analogy, the prefrontal cortex may invoke a variety of gating or filtering devices in its role in monitoring and controlling information processing. It is proposed here that the prefrontal cortex operates as a dynamic filtering mechanism that maintains selected neural activations and gates extraneous or irrelevant ones. According to this view, at any given moment cortical and subcortical activations involved in sensory and cognitive functioning produce a cacophony of neural signals. The prefrontal cortex, with 
its extensive projections to and from many cortical and subcortical regions, orchestrates these signals by means of a filtering mechanism that inhibits some signals and maintains activation of others. In essence, the prefrontal cortex acts to refine activity and increase signal-to-noise ratios. This mechanism may be particularly involved in inhibiting or damping extraneous activity, or "noise," under conditions of extensive interference (for related views, see Cohen \& Servan-Schreiber, 1992; Desimone \& Duncan, 1995).

The prefrontal cortex is extensively connected to many posterior cortical regions via reciprocal projections ( $\mathrm{Pan}$ dya \& Yeterian, 1998). These connections maintain some segregation such that projections from a particular posterior region will project to a specific region in the prefrontal cortex (Wilson et al., 1993). Dynamic filtering theory suggests that activation in the posterior cortex initiates a pattern of associated activations in the prefrontal cortex. By way of reciprocal projections back to the posterior regions, this pattern of activation enables certain neuronal ensembles to be kept active and others to be gated or inhibited. This mechanism affords a means by which information processing in the posterior cortex can be maintained and modulated by signals from the prefrontal cortex. Without prefrontal control, the system is subject to greater noise from extraneous activations.

Given this neural architecture, implementation of control can occur in several ways. The most basic control may be based simply on the state of neural activity at any given moment. In particular, the prefrontal cortex may initiate some control by inhibiting regions that have just been activated. That is, efficient temporal processing may require the clipping of recently active information as a way to segregate neural processing from one moment to another. The prefrontal cortex might control neural activity by simply reflecting an inhibitory signal back to the same posterior regions that have recently been active. This form of control amounts to a neural "inhibition of return," which could facilitate attentional shifts. Without this simple control, the system might be subject to perseverations of recent activations.

Another way to implement control is to associate specific filters with specific memory representations. Perhaps through experience, we acquire not only a vast amount of knowledge but also a multitude of filters that enable the selection of that knowledge. For example, a cue, such as the word animals or psychobiology, would be associated not only with a distributed network of knowledge, but also with numerous filters associated with that knowledge. These filters, represented as patterns of activation in the prefrontal cortex, would then be implemented in response to specific cues. Thus, the word animals would not only activate representations in posterior cortical areas but also activate filters in the prefrontal cortex, which then would select, maintain, and modulate associated posterior activations. In addition to these acquired filters, it is possible that there exist innate filters that enable selection of sensory modalities and motor programs.

When prefrontal function is viewed as a dynamic filtering mechanism, executive control processes can be interpreted as aspects of filtering. Table 1 is an outline of the four executive control processes reviewed here-selecting, maintaining, updating, and rerouting-which defines an aspect of filtering for each one. In dynamic filtering theory, selecting involves the engagement or initial selection of a filter. Maintaining involves filter persistence or the ability to keep a filter fixed across time. Filter persistence is paramount for short-term memory tasks, such as digit span. Updating involves shifting activation from one stimulus-based activation to another. That is, certain filters are tied closely to sensory modalities, and these filters enable the switching from one stimulus to another. Rerouting requires greater amounts of filter shifting because entire processing streams, including response programs, must be disengaged and rerouted. Thus, rerouting involves filter switching based on shifts of processing associated with task sets and response programs. In addition to relating different aspects of filtering to aspects of executive control, it may also be that levels of control, from selecting to rerouting, engage increasingly more complex representations. Thus, aspects of selecting may involve modality-specific processing (e.g., sensory processes), whereas updating and rerouting involve polymodal processing (for related views, see D'Esposito \& Postle, in press; Petrides, 1998).

Dynamic filtering theory suggests that prefrontal activity engages both selection and active inhibition of posterior cortical areas. The notion of selection is less controversial than the notion of active inhibition. However, some physiological evidence suggests that the prefrontal cortex engages inhibitory control of posterior cortical activity. Knight and colleagues (Knight, Scabini, \& Woods, 1989; Yamaguchi \& Knight, 1990) studied event-related potentials (ERPs) in patients with dorsolateral prefrontal

Table 1

Aspects of Executive Control and Dynamic Filtering Theory

\begin{tabular}{|c|c|c|c|}
\hline $\begin{array}{l}\text { Executive Control } \\
\text { Process }\end{array}$ & $\begin{array}{l}\text { Related } \\
\text { Concept }\end{array}$ & $\begin{array}{l}\text { Benchmark } \\
\text { Task }\end{array}$ & $\begin{array}{c}\text { Filtering } \\
\text { Mechanism }\end{array}$ \\
\hline \multicolumn{4}{|l|}{ Activation } \\
\hline Selecting & Selective attention & Stroop & Filter selection \\
\hline Maintaining & Short-term memory & Digit span & Filter persistence \\
\hline \multicolumn{4}{|l|}{ Manipulation } \\
\hline Updating & Monitoring & $n$-back & Filter switch-stimulus \\
\hline Rerouting & Set shifting & Task switching & Filter switch-response \\
\hline
\end{tabular}


lesions. In one study (Knight et al., 1989), the amplitude of evoked responses presumed to be generated in the primary auditory cortex (i.e., middle latency evoked potentials) was potentiated in patients with prefrontal lesions. Thus, there appeared to be a disinhibition of posterior cortical activity as a result of a frontal lobe lesion. Control of unimodal sensory areas could be mediated by cortical or subcortical connections. Interestingly, an inhibitory control mechanism has been proposed for frontal control via thalamic connections. Specifically, it has been suggested that frontal projections to the dorsomedial nucleus of the thalamus modulate thalamic activity by an inhibitory mechanism (Skinner \& Yingling, 1977). This inhibitory control mechanism may filter or gate sensory information at very early stages of processing. Such modulations could be implemented by direct inhibitory connections to target areas or, indirectly, by excitatory connections to inhibitory neurons (e.g., interneurons) in target areas.

In a PET study of normal individuals engaged in cognitive tasks (Frith et al., 1991), an increase in activity in the dorsolateral prefrontal cortex was related to a decrease in activity in posterior cortical regions. These findings fit well with the notion that increases in dynamic filtering cause more refined and sometimes an overall decrease in posterior activity in certain regions. Recently, Konishi et al. (1999) used event-related fMRIs to study activity during a go/no-go task. Right (inferior) dorsolateral prefrontal activation was associated with no-go trials, and the authors suggest that this activation serves as an inhibitory signal. Interestingly, the same prefrontal region was active when the subjects performed the Wisconsin Card Sorting test, suggesting that task shifting may be related to inhibitory control mechanisms.

On the basis of dynamic filtering theory, the multitude of cognitive disorders observed in patients with prefrontal lesions can be explained by the failure of filtering activity in the posterior cortex. However, this view does not imply a unitary central executive system. Indeed, it is known that different regions within the prefrontal cortex affect different aspects of cognition. It is proposed here that the variety of dysfunctions associated with prefrontal lesions can be attributed to differences in regional specificity in the anatomical connections from prefrontal regions to areas in posterior cortex. That is, prefrontal regions are performing the same neural functiondynamic filtering - but different behavioral outcomes occur because different prefrontal regions are filtering different posterior cortical regions, which themselves serve different cognitive functions. According to this view, damage to a specific region in the prefrontal cortex will affect executive control (i.e., dynamic filtering) of a specific function defined by the areas in the posterior cortex to which that prefrontal region is anatomically connected.

Filtering problems may occur at many levels of information processing, including sensory processing, memory activation, and response selection. At the level of sensory processing, prefrontal (and anterior cingulate) function may involve the selection and maintenance of sensory signals. According to dynamic filtering theory, prefrontal regions will be involved in controlling sensory processes to the extent that these regions are connected to posterior cortical regions important for sensory processing. Other prefrontal regions may be involved in the filtering of conceptual information. Such areas would control the maintenance and updating of information in memory. Demands on dynamic filtering will occur to the extent that irrelevant activations intrude on information processing, such as in tasks involving dominant responses that must be inhibited. In fact, the ability to inhibit previously dominant memory activations (i.e., overcoming proactive interference) is an important feature of prefrontal control (Dolan \& Fletcher, 1997; Jonides, Smith, Marshuetz, Koeppe, \& Reuter-Lorenz, 1998; Shimamura, Jurica, Mangels, Gershberg, \& Knight, 1995). Because rerouting depends on the shifting of many cognitive processes, including response mappings, this function may depend on different or more extensive filtering. In particular, rerouting may involve prefrontal regions that are tied to cortical regions important for response programming and initiation. Thus, dynamic filtering is a unifying mechanism of prefrontal activity, but its expression in performance depends on the brain regions that are being controlled.

\section{EMOTIONAL CONTROL AND ORBITOFRONTAL CORTEX}

Dynamic filtering theory was initially developed in order to characterize the role of the dorsolateral prefrontal cortex in cognition and memory (Shimamura, 1994, 1996). Yet this mechanism may be useful as a way of characterizing the role of the orbitofrontal cortex in filtering and controlling emotions. Ever since John Martyn Harlow's description of Phineas Gage (Harlow, 1848, 1868; see also Macmillan, 1986, 2000), the orbitotfrontal cortex has been linked to the regulation and control of emotions. As is well known, Gage's emotional disposition changed following a bizarre head injury in which an iron rod penetrated, harpoon-like, through his skull, damaging his orbitofrontal cortex. Harlow examined and treated Gage after his accident. In writing about this case, Harlow (1868) describes Gage's disposition:

$\mathrm{He}$ is fitful, irreverent, indulging at times in the grossest profanity (which was not previously his custom), manifesting but little deference for his fellows, impatient of restraint or advice when it conflicts with his desires, at times pertinaciously obstinate, yet capricious and vacillating, devising many plans of future operation, which are sooner arranged than that are abandoned in turn for others appearing more feasible ... In this regard his mind was radically changed, so decidedly that his friends and acquaintances said he was "no longer Gage."

The description of Gage's affective disorder is familiar to neurologists who treat patients with orbitofrontal damage. Orbitofrontal damage and emotional disinhibition are frequent consequences of severe traumatic head injury, 
such as those incurred following automobile accidents (Mattson \& Levin, 1990). It is also observed in a dementing disorder called frontotemporal dementia (Miller, Darby, Swartz, Yener, \& Mena, 1995). In cases of orbitofrontal damage, symptoms include emotional outbursts, social disinhibition, impulsivity, risk-taking, and obsessivecompulsive behavior (see Damasio, 1995; Lhermitte, 1986; Shimamura, 2000; Stuss \& Benson, 1986). On the basis of dynamic filtering theory, these symptoms can be attributed to a failure to regulate or gate posterior cortical (and likely subcortical) regions involved in the expression of emotions.

Rule, Shimamura, and Knight (1999) recently obtained electrophysiological evidence of neural disinhibition in patients with orbitofrontal damage. ERPs were recorded in response to mild wrist shocks and abrupt auditory stimuli. These stimuli typically elicit an orienting response that habituates after repeated presentations. Patients with orbitofrontal lesions exhibited heightened ERP amplitudes in response to these stimuli, in comparison with neurologically intact control subjects and with patients with dorsolateral prefrontal lesions. Also, patients with orbitofrontal lesions were less likely to habituate to these stimuli after repeated presentations. The source of these ERPs appeared to be located in posterior cortical regions. Thus, orbitofrontal lesions produced a disinhibition of neural activity in response to affectively laden stimuli.

\section{DYNAMIC FILTERING THEORY IN RELATION TO OTHER VIEWS}

Dynamic filtering theory offers a neural-based mechanism that enables top-down control of information processing. This view builds on earlier theories of prefrontal function. Previous views have emphasized different aspects of executive control. Baddeley's characterization of working memory and the central executive (Baddeley, 1986) emphasizes activation and maintenance of information in short-term memory. Goldman-Rakic (1987, 1998 ) and Fuster (1989) offered empirical findings and neural-based theories of short-term memory activation and maintenance. Dynamic filtering theory is consistent with these views in suggesting that the prefrontal cortex is involved in selecting and maintaining activation in short-term memory. Specifically, the prefrontal cortex is presumed to be involved with filter selection and filter persistence.

Goldman-Rakic (1998) suggests that regional specificity within the prefrontal cortex is due to differences in the kind of information being processed (e.g., spatial vs. object information) rather than to the way in which information is being processed. For Goldman-Rakic (1998), prefrontal mechanisms involve the active maintenance of specific memory representations in the absence of sensory-driven activation. In dynamic filtering theory, regional specificity is also presumed to be driven by the kind of information modulated. However, dynamic filtering theory uses the same mechanism to account for more complex aspects of executive control, such as updating and rerouting. Indeed, depending on the kind of activity that is filtered (e.g., sensory, memory, task sets, response programs, or emotions), more complex executive control can be implemented.

Tulving (Tulving, Kapur, Craik, Moscovitch, \& Houle, 1994) proposed a model of frontal lobe function characterized by differential processing of information in the two cerebral hemispheres (for review, see Nyberg et al., 1996). In this model, called HERA (hemispheric encoding/retrieval asymmetry), the left prefrontal cortex is presumed to be more involved with semantic encoding, whereas the right prefrontal cortex is more involved with episodic retrieval. This model is based on neuroimaging findings of hemisphere asymmetries in processing semantic and episodic information. However, it has been suggested that these effects may be better characterized by regional specificity of prefrontal function. For example, Buckner (1996) has suggested that a more detailed view would consider hemispheric asymmetries to be related to differential executive control of verbal and nonverbal information (see also Smith \& Jonides, 1999). Such a view is more consistent with studies of patients with unilateral lesions. According to the dynamic filtering theory, the left and right prefrontal cortices are both engaged in filtering operations. Behavioral effects will differ depending on the kind of information that is being filtered. It is likely that left and right prefrontal regions filter different aspects of information processing. In general, left prefrontal regions are likely involved with filtering lexical/semantic/featural representations, whereas right prefrontal regions are likely involved with filtering spatial/episodic/global representations.

To account for the role of the prefrontal cortex in both maintaining and manipulating information, others have suggested that posterior cortical activity can be modulated by prefrontal activity. For example, Knight, Staines, Swick, and Chao (1999) and Miller et al. (1996) have suggested that prefrontal activity enables executive control by selecting or inhibiting activity in posterior regions. This kind of neural mechanism is similar to that proposed by dynamic filtering theory. To account for aspects of executive control, Petrides $(1994,1998)$ suggests a two-level framework in which mid-ventrolateral and mid-dorsolateral regions of the prefrontal cortex perform different operations. Mid-ventrolateral regions are presumed to be involved in first-order executive control processes associated with selection and maintenance of short-term memory. Mid-dorsolateral regions act as second-order executive processes involved in monitoring and manipulation. Related descriptions of multiple levels of executive control have been discussed by others (see D'Esposito, Aguirre, Zarahn, \& Ballard, 1998; Smith \& Jonides, 1999).

Petrides $(1994,1998)$ has suggested that mid-ventrolateral and mid-dorsal regions process information in different ways. Thus, unlike Goldman-Rakic and others (Buckner, 1996; Goldman-Rakic, 1987; Smith \& Jonides, 1999), Petrides has suggested that regional specificity is 
due to differences in the manner in which information is processed. By invoking qualitatively different processes for different prefrontal regions, this view is also unlike dynamic filtering theory. Although dynamic filtering theory suggests a unified neural mechanism within the prefrontal cortex, what is being filtered determines the way in which information is processed. For example, the filtering of stimulus features facilitates perceptual analysis and selective attention. The filtering of memory activations facilitates memory organization and retrieval. The filtering of response programs facilitates task switching and avoids perseveration of central sets. The filtering of emotional responses facilitates behavioral control in social interactions. Thus, selecting, maintaining, updating, rerouting, and even emotion regulation could be implemented by different regions in the prefrontal cortex, even though the same filtering mechanism is being invoked.

In the model proposed by Petrides (1998), prefrontal function is divided into two separate functions. It is suggested that these two functions are organized hierarchically, with mid-dorsolateral regions acting at a higher level than mid-ventrolateral regions do. Dynamic filtering theory suggests that there are at least four levels of executive control-selecting, maintaining, updating, and rerouting (see Table 1). These aspects form the four focal categories of executive control. However, there may be even further, more detailed ways to define the manner in which filtering can affect information processing.

Several computational models of prefrontal function have been developed. Kimberg and Farah (1993) have proposed a model in which executive control is imposed by affecting links that associate information in working memory. These links permit selection of appropriate items in working memory within a contextual framework. For example, in the Stroop test, the contextual factor involves responding to color and not to color names. Without such associative links, performance is less contingent on topdown (i.e., contextual) control, and problems arise that simulate problems associated with frontal lobe dysfunction. For example, this computational model can account for problems on the Wisconsin Card Sort test, Stroop test, and other tests that involve top-down control of stimulus selection. Cohen and Servan-Schreiber (1992) have offered a similar computational model in which context setting is viewed as the primary feature of selective attention. As Cohen and Servan-Schreiber (p. 46) stated, "By this definition, context information is relevant to but does not form part of the content of the actual response."

In the models proposed by Cohen and Servan-Schreiber (1992) and Kimberg and Farah (1993), executive control is implemented by reinforcing task-relevant processes or associations (i.e., context setting). Such models can be viewed as refining information processing by heightening or selecting appropriate information processing. Considerable success in modeling executive control and frontal lobe function has been attained by this kind of mechanism. On the other hand, here I have emphasized the importance of both selection and active inhibition as mech- anisms of filter control (see also Knight et al., 1999; Shimamura, 1994). As demonstrated by these computational models, implementation of an inhibitory control mechanism does not appear to be necessary to account for aspects of frontal lobe function. Also, in terms of behavioral outcome, it is extremely difficult to differentiate a model based purely on selection from a model based on both selection and inhibition. In the end, the two models can often lead to similar behavioral outcomes.

One computational model that implements both selection and inhibitory control is Grossberg's $(1982,1999)$ general model of neural control called adaptive resonance theory. In this model, control is implemented by a topdown filtering mechanism that enhances task-relevant information in working memory and inhibits similar but irrelevant information. This kind of filtering is analogous to center-on, surround-off receptive fields. That is, cortical processing is sharpened by both the enhancement of relevant activity (center-on) and inhibition of irrelevant activity (surround-off). Although this mechanism has been applied as a control of neural processing at the micro-level, on a larger scale, the prefrontal cortex may implement such a dynamic filtering mechanism more globally in order to control information processing not amenable by interactions within local circuits. By analogy, the kinds of lateral inhibition effects observed in visual processing may occur for neural systems involved in memory representation or motor programming. Although such processes can be assumed to occur in local neural circuits, the prefrontal cortex may also initiate such filtering at more macro levels. Grossberg's computational model most closely characterizes the dynamic filtering mechanism proposed here.

\section{CONCLUSION}

In summary, the notion of dynamic filtering can be a useful way to describe many if not most aspects of prefrontal function. Findings from patients with frontal lobe lesions and from functional neuroimaging studies suggest that the prefrontal cortex is involved in selecting appropriate processing modes and filtering irrelevant or extraneous ones. Indeed, many aspects of executive control, such as selecting, maintaining, updating, and rerouting information processing, could be interpreted as aspects of dynamic filtering, such as filter selection, filter persistence, and filter switching. Importantly, a variety of experimental techniques (e.g., behavioral, physiological, and anatomical) can be used to assess predictions based on dynamic filtering because the theory describes a neural-based model that enables executive control.

\section{REFERENCES}

Allport, D. A., Styles, E. A., \& Hsieh, S. (1994). Shifting intentional set: Exploring the dynamic control of tasks. In C. Umiltà \& M. Moscovitch (Eds.), Attention and performance $X V$ : Conscious and nonconscious information processing (pp. 421-452). Cambridge, MA: MIT Press. 
AWh, E., Jonides, J., Smith, E. E., Schumacher, E. H., \& Koeppe, R. A. (1996). Dissociation of storage and rehearsal in verbal working memory: Evidence from positron emission tomography. Psychological Science, 7, 25-31.

Baddeley, A. (1986). Working memory. Oxford: Oxford University Press.

Baldo, J. V., \& Shimamura, A. P. (1995, April). Performance of frontal lobe patients on Stroop-like tasks. Paper presented at the annual meeting of the Cognitive Neuroscience Society, San Francisco.

Baldo, J. V., \& ShimamuRA, A. P. (1998). Letter and category fluency in patients with frontal lobe lesions. Neuropsychology, 12, 259-267.

Baldo, J. V., \& Shimamura, A. P. (2000). Spatial and color working memory in patients with lateral prefrontal cortex lesions. Psychobi$o \log y, 28,156-167$.

Bench, C. J., Frith, C. D., Grasby, P. M., \& Friston, K. J. (1993). Investigations of the functional anatomy of attention using the Stroop test. Neuropsychologia, 31, 907-922.

Benton, A., \& DEHAMSHER, K. (1976). Multilingual aphasia examination. Iowa City: University of Iowa Press.

Bonin, G., \& BaILY, P. (1947). The neocortex of the Macaca mulatta. Urbana: University of Illinois Press.

Botvinick, M., Nystrom, E., Fissell, K., \& Carter, C. S. (1999). Conflict monitoring versus selection-for-action in anterior cingulate cortex. Nature, 402, 179-181.

Brewer, J. B., Zhao, Z., Desmond, J. E., Glover, G. H., \& Gabrieli, J. D. E. (1998). Making memories: Brain activity that predicts how well visual experience will be remembered. Science, 281, 1185-1187.

BUCKNER, R. L. (1996). Beyond HERA: Contributions of specific prefrontal brain areas to long-term memory retrieval. Psychonomic Bulletin \& Review, 3, 149-158.

Carlson, S., Martinkauppi, S., Raemae, P., \& Salli, E. (1998). Distribution of cortical activation during visuospatial $n$-back tasks as revealed by functional magnetic resonance imaging. Cerebral Cortex, 8, 743-752.

ChaO, L. L., \& Knight, R. T. (1996). Prefrontal and posterior cortical activation during auditory working memory. Cognitive Brain Research, 4, 27-37.

Cohen, J. D., Perlstein, W. M., Braver, T. S., \& Nystrom, L. E. (1997). Temporal dynamics of brain activation during a working memory task. Nature, 386, 604-608.

Cohen, J. D., \& Servan-Schreiber, D. (1992). Context, cortex, and dopamine: A connectionist approach to behavior and biology in schizophrenia. Psychological Review, 99, 45-77.

DAMASIO, A. (1995). On some functions of the human prefrontal cortex. In J. Grafman, K. J. Holyoak, \& F. Boller (Eds.), Structure and function of the human prefrontal cortex (Annals of the New York Academy of Sciences, Vol. 769, pp. 241-251). New York: New York Academy of Sciences.

Delis, D. C., Squire, L. R., Bihrle, A., \& Massman, P. (1992). Componential analysis of problem-solving ability: Performance of patients with frontal lobe damage and amnesic patients on a new sorting test. Neuropsychologia, 30, 683-697.

Demb, J. B., Desmond, J. E., Wagner, A. D., Vaidya, C. J., Glover, G. H., \& GABRIELI, J. D. E. (1995). Semantic encoding and retrieval in the left inferior prefrontal cortex: A functional MRI study of task difficulty and process specificity. Journal of Neuroscience, 15, 58705878.

Desimone, R., \& DunCan, J. (1995). Neural mechanisms of selective visual attention. Annual Review of Neuroscience, 18, 193-222.

D'Esposito, M., Aguirre, G. K., Zarahn, E., \& Ballard, D. (1998). Functional MRI studies of spatial and nonspatial working memory. Cognitive Brain Research, 7, 1-13.

D'Esposito, M., Detre, J. A., Alsop, D. C., \& Shin, R. K. (1995). The neural basis of the central executive system of working memory. $\mathrm{Na}$ ture, 1378, 279-281.

D'Esposito, M., \& Postle, B. R. (1999). The dependence of span and delayed-response performance on prefrontal cortex. Neuropsychologia, 37, 1303-1315.

D'Esposiro, M., \& PostLE, B. R. (in press). Neural correlates of component processes of working memory: Evidence from neuropsychological and pharmocological studies. In S. Monsell \& J. Driver (Eds.),
Attention and performance XVIII: Control of cognitive processes. Cambridge, MA: MIT Press.

Dias, R., Robbins, T. W., \& Roberts, A. C. (1996). Dissociation in prefrontal cortex of affective and attentional shifts. Nature, 380, 69-72.

Dolan, R. J., \& Fletcher, P. C. (1997). Dissociating prefrontal and hippocampal function in episodic memory encoding. Nature, $\mathbf{3 8 8}$, 582-585.

DUNBar, K., \& SusSman, D. (1995). Toward a cognitive account of frontal lobe function: Simulating frontal lobe deficits in normal subjects. In J. Grafman, K. J. Holyoak, \& F. Boller (Eds.), Structure and functions of the human prefrontal cortex (Annals of the New York Academy of Sciences, Vol. 769, pp. 289-304). New York: New York Academy of Sciences.

Duncan, J., Emslie, H., Williams, P., \& Johnson, R. (1996). Intelligence and the frontal lobe: The organization of goal-directed behavior. Cognitive Psychology, 30, 257-303.

EsLiNGER, P. J., \& GRATTAN, L. M. (1994). Altered serial position learning after frontal lobe lesion. Neuropsychologia, 32, 729-739.

Frith, C., Friston, K., Liddle, P., \& Frackowiak, D. (1991). A PET study of word finding. Neuropsychologia, 29, 1137-1148.

Funahashi, S., Bruce, C. J., \& Goldman-Rakic, P. S. (1993). Dorsolateral prefrontal lesions and oculomotor delayed-response performance: Evidence for mnemonic "scotomas." Journal of Neurascience, 13, 1479-1497.

Fuster, J. M. (1989). The prefrontal cortex (2nd ed.). New York: Raven Press.

Gabrieli, J. D., Desmond, J. E., Demb, J. B., Wagner, A. D., Stone, M. V., VAIDYA, C. J., \& Glover, G. H. (1996). Functional magnetic resonance imaging of semantic memory processes in the frontal lobes. Psychological Science, 7, 278-283.

GershberG, F. B., \& Shimamura, A. P. (1995). The role of the frontal lobes in the use of organizational strategies in free recall. Neuropsychologia, 13, 1395-1333.

Goldman-RAKIC, P. S. (1987). Circuitry of primate prefrontal cortex and regulation of behavior by representational memory. In F. Plum \& V. B. Mountcastle (Eds.), Handbook of Physiology: Sec. I. The nervous system: Vol. 5. Higher functions of the brain (pp. 373-417). Bethesda, MD: American Physiological Society.

GoldMAN-RAKIC, P. (1998). The prefrontal landscape: Implications of functional architecture for understanding human mentation and the central executive. In A. C. Roberts, T. W. Robbins, \& L. Weiskrantz (Eds.), The prefrontal cortex: Executive and cognitive function (pp. 87-102). Oxford: Oxford University Press.

GoldSTEIN, K. (1936). The significance of the frontal lobes for mental performance. Journal of Neurology \& Psychopathology, 17, 27-40.

GROSSBERG, S. (1982). Studies of mind and brain: Neural principles of learning, perception, development, cognition, and motor control. Norwell, MA: Kluwer.

GrossBerg, S. (1999). The link between brain learning, attention, and consciousness. Consciousness \& Cognition, 8, 1-44.

HALSTEAD, W. C. (1947). Brain and intelligence: A quantitative study of the frontal lobes. Chicago: University of Chicago Press.

HARLOW, J. M. (1848). Passage of an iron rod through the head. Boston Medical \& Surgical Journal, 39, 389-393.

HaRLow, J. M. (1868). Recovery of an iron rod through the head. Publication of the Massachusetts Medical Society, 2, 327-347.

HebB, D. O. (1945). Man's frontal lobes: A critical review. Archives of Neurology \& Psychiatry, 54, 10-24.

HirsT, W., \& VolPE, B. T. (1988). Memory strategies with brain damage. Brain \& Cognition, 8, 379-408.

JACOBSON, C. F. (1936). Studies of cerebral function in primates. Comparative Psychology Monographs, 13, 1-8.

Janowsky, J. S., Shimamura, A. P., Kritchevsky, M., \& Squire, L. R. (1989). Cognitive impairment following frontal lobe damage and its relevance to human amnesia. Behavioral Neuroscience, 103, 548-560.

Janowsky, J. S., Shimamura, A. P., \& SQuire, L. R. (1989). Source memory impairment in patients with frontal lobe lesions. Neuropsychologia, 27, 1043-1056.

Johnson, M. K., Hashtroudi, S., \& Lindsay, D. S. (1993). Source monitoring. Psychological Bulletin, 114, 3-28. 
Jones-Gotman, M., \& Milner, B. (1977). Design fluency: The invention of nonsense drawings after focal cortical lesion. Neuropsychologia, 15, 653-674.

Jonides, J., Schumacher, E. H., Smith, E. E., Lauber, E. J., Awh, E., Minoshima, S., \& Koeppe, R. A. (1997). Verbal working memory load affects regional brain activation as measured by PET. Journal of Cognitive Neuroscience, 9, 462-475.

Jonides, J., Smith, E. E., Koeppe, R. A., Awh, E., Minoshima, S., \& Mintun, M. A. (1993). Spatial working memory in humans as revealed by PET. Nature, 363, 623-625.

Jonides, J., Smith, E. E., Marshuetz, C., Koeppe, R. A., \& ReuterLORENZ, P. A. (1998). Inhibition of verbal working memory revealed by brain activation. Proceedings of the National Academy of Sciences, 95, 8410-8413.

KIMBERG, D. Y., \& FARAH, M. J. (1993). A unified account of cognitive impairments following frontal lobe damage: The role of working memory in complex, organized behavior. Journal of Experimental Psychology: General, 122, 411-428.

KNight, R. T., Scabini, D., \& Woods, D. L. (1989). Prefrontal gating of auditory transmission in humans. Brain Research, 504, 338-342.

Knight, R. T., Staines, W. R., Swick, D., \& Chao, L. L. (1999). Prefrontal cortex regulates inhibition and excitation in distributed neural networks. Acta Psychologica, 101, 159-178.

Konishi, S., Nakajima, K., Uchida, I., Kikyo, H., Kameyama, M., \& MiYashitA, Y. (1999). Common inhibitory mechanism in human inferior prefrontal cortex revealed by event-related functional MRI. Brain, 122, 981-991.

LhermitTe, F. (1986). Human autonomy and the frontal lobes: Part II. Patient behavior in complex and social situations: The "environmental dependency syndrome." Annals of Neurology, 19, 335-343.

LuRIA, A. R. (1966). Higher cortical functions in man. New York: Basic Books.

MACLEOD, C. (1991). Half a century of research on the Stroop effect: An integrative review. Psychological Bulletin, 109, 163-203.

MaCmillaN, M. B. (1986). A wonderful journey through skull and brains: The travels of Mr. Gage's tamping iron. Brain \& Cognition, 5, 67-107.

Macmillan, M. B. (2000). An odd kind of fame: Stories of Phineas Gage. Cambridge, MA: MIT Press.

MatTson, A. J., \& Levin, H. S. (1990). Frontal lobe dysfunction following closed head injury. Journal of Nervous \& Mental Disease, 178, 282-291.

McCarthy, G., Blamire, A. M., Rothman, D. L., Gruetter, R., \& Shulman, R. G. (1993). Echo-planar magnetic resonance imaging studies of frontal cortex activation during word generation in humans. Proceedings of the National Academy of Sciences, 90, 4952-4956.

MilleR, B. L., \& Cummings, J. L. (EDs.) (1999). The human frontal lobes: Functions and disorders. New York: Guilford

Miller, B. L., Darby, A. L., Swartz, J. R., Yener, G. G., \& Mena, I. (1995). Dietary changes, compulsions and sexual behavior in frontotemporal degeneration. Dementia, 6, 195-199.

Miller, E. K., Erickson, C. A., \& Desimone, R. (1996). Neural mechanisms of visual working memory in prefrontal cortex of the macaque. Journal of Neuroscience, 16, 5154-5167.

MiLNER, B. (1964). Some effects of frontal lobectomy in man. In J. Warren \& $\mathrm{K}$. Akert (Eds.), The frontal granular cortex and behavior (pp. 313-331). New York: McGraw-Hill.

MILNER, B. (1971). Interhemispheric differences in the localization of psychological processes in man. British Medical Bulletin, 127, 272-277.

Milner, B., Petrides, M., \& Smith, M. L. (1985). Frontal lobes and the temporal organization of memory. Human Neurobiology, 4, 137-142.

MishKIN, M. (1964). Perseveration of central sets after frontal lesions in monkey. In J. M. Warren \& K. Akert (Eds.), The frontal granular cortex and behavior (pp. 219-241), New York: McGraw-Hill.

Mishkin, M., \& MaNNING, F. J. (1978). Nonspatial memory after selective prefrontal lesions in monkeys. Brain Research, 143, 313-323.

MonSELl, S. (1996). Control of mental processes. In V. Bruce (Ed.), Unsolved mysteries of the mind: Tutorial essays in cognition (pp. 93148). Hove, U.K.: Erlbaum Taylor \& Francis.

Moscovitch, M. (1994). Cognitive resources and dual-task interference effects at retrieval in normal people: The role of the frontal lobes and medial temporal cortex. Neuropsychology, 8, 524-534.

Nolde, S. F., Johnson, M. K., \& D'Esposito, M. (1998). Left prefrontal activation during episodic remembering: An event-related fMRI study. NeuroReport, 9, 3509-3514.

Nyberg, L., Cabeza, R., \& Tulving, E. (1996). PET studies of encoding and retrieval: The HERA model. Psychonomic Bulletin \& Review, 3, 135-148.

Owen, A. M., Downes, J. J., Sahakian, B. J., Polkey, C. E., \& RobBINS, T. W. (1990). Planning and spatial working memory following frontal lobe lesions in man. Neuropsychologia, 28, 1021-1034.

Owen, A. M., Roberts, A. C., Hodges, J. R., Summers, B. A., Polkey C. E., \& RoBBins, T. W. (1993). Contrasting mechanisms of impaired attentional set-shifting in patients with frontal lobe damage or Parkinson's disease. Brain, 116, 1159-1175.

Pandya, D. N., \& Yeterian, E. H. (1998). Comparison of prefrontal architecture and connections. In A. C. Roberts, T. W. Robbins, \& L. Weiskrantz (Eds.) The prefrontal cortex (pp. 51-66). Oxford: Oxford University Press.

PERrET, E. (1974). The left frontal lobe of man and the suppression of habitual responses in verbal categorical behaviour. Neuropsychologia, 12, 323-330.

Peterson, B. S., Bradley S., Skudlarski, P., Gatenby, J. C., \& ZHANG, H. (1999). An fMRI study of Stroop word-color interference: Evidence for cingulate subregions subserving multiple distributed attentional systems. Biological Psychiatry, 45, 1237-1258.

Petrides, M. (1994). Frontal lobes and working memory: Evidence from investigations of the effects of cortical excisions in nonhuman primates. In F. Boller \& J. Grafman (Eds.), Handbook of neuropsychology (Vol. 9, pp. 75-90). Amsterdam: Elsevier.

Petrides, M. (1998). Specialized systems for the processing of mnemonic information within the primate frontal cortex. In A. C. Roberts, T. W. Robbins, \& L. Weiskrantz (Eds.), The prefrontal cortex: Executive and cognitive function (pp. 103-116). Oxford: Oxford University Press.

Petrides, M., Alivisatos, B., \& Evans, A. C. (1995). Functional activation of the human ventrolateral frontal cortex during mnemonic retrieval of verbal information. Proceedings of the National Academy of Sciences, 92, 5803-5807.

Petrides, M., Alivisatos, B., Evans, A. C., \& Meyer, E. (1993). Dissociation of human mid-dorsolateral frontal cortex in memory processing. Proceedings of the National Academy of Sciences, 90, 878-882.

Petrides, M., \& MiLner, B. (1982). Deficits on subject-ordered tasks after frontal- and temporal-lobe lesions in man. Neuropsychologia, 20, 249-262.

Petrides, M., \& Pandya, D. N. (1994). Comparative architectonic analysis of the human and macaque frontal cortex. In F. Boller \& J. Grafman (Eds.), Handbook of neuropsychology (Vol. 9, pp. 59-82). Amsterdam: Elsevier.

Ptito, A., Crane, J., Leonard, G., Amsel, R., \& Caramanos, Z. (1995). Visual-spatial localization by patients with frontal-lobe lesions invading or sparing area 46. NeuroReport, 6, 1781-1784.

Rafal, R., GershberG, F., Egly, R., \& IVRY, R. (1996). Response channel activation and the lateral prefrontal cortex. Neuropsychologia, 34, 1197-1202.

Rainer, G., AsaAD, W. F., \& Miller, E. K. (1998). Selective representation of relevant information by neurons in the primate prefrontal cortex. Nature, 393, 577-579.

RAO, S. C., RAINER, G., \& Miller, E. K. (1997). Integration of what and where in the primate prefrontal cortex. Science, 276, 821-824.

Roberts, A. C., Robiins, T. W., \& Weiskrantz, L. (Eds.) (1998). The prefrontal cortex: Executive and cognitive function. Oxford: Oxford University Press.

Rule, R., Shimamura, A. P., \& KNight, R. P. (1999, November). Disinhibition of emotional responses following lesions of the ventromedial prefrontal cortex. Paper presented at the Society for Neuroscience Annual Meeting.

Schacter, D. L., Harbluk, J., \& Mclachlan, D. (1984). Retrieval without recollection: An expert analysis of source amnesia. Journal of Verbal Learning \& Verbal Behavior, 23, 593-611.

Shallice, T. (1982). Specific impairments in planning. Philosophical Transactions of the Royal Society of London: Series B, 298, 199-209. 
Shimamura, A. P. (1994). Memory and frontal lobe function. In M. S Gazzaniga (Ed.), The cognitive neurosciences (pp. 803-813), Cambridge, MA: MIT Press.

Shimamura, A. P. (1996). The control and monitoring of memory functions. In L. Reder (Ed.), Metacognition and implicit memory (pp. 259274). Mahwah, NJ: Erlbaum.

Shimamura, A. P. (2000). The neurological case of Eadweard J. Muybridge: The role of the orbitofrontal cortex in regulating emotions. Manuscript submitted for publication.

Shimamura, A. P., Janowsky, J. S., \&. SQUiRe, L. R. (1990). Memory for the temporal order of events in patients with frontal lobe lesions and amnesic patients. Neuropsychologia, 28, 803-813.

Shimamura, A. P., Jurica, P. J., Mangels, J. A., Gershberg, F. B., \& KNIGHT, R. T. (1995). Susceptibility to memory interference effects following frontal lobe damage: Findings from tests of paired-associated learning. Journal of Cognitive Neuroscience, 7, 144-152.

SkINNER, J. E., \& YINGLING, C. D. (1977). Central gating mechanisms that regulate event-related potentials and behavior. In J. E. Desmedt (Ed.), Attention, voluntary contraction, and event-related cerebral potentials (Progress in Clinical Neurophysiology, Vol. 1, pp. 30-69). Basel: Karger.

Smith, E. E., \& Jonides, J. (1999). Storage and executive processes in the frontal lobes. Science, 283, 1657-1661.

Smith, E. E., Jonides, J., KoepPe, R. A., AWh, E., Schumacher, E. H., \& Minoshima, S. (1995). Spatial versus object working memory: PET investigations. Journal of Cognitive Neuroscience, 7, 337-356.

STROOP, J. (1935). Studies of interference in serial verbal reactions. Journal of Experimental Psychology, 18, 643-662.

STuss, D. T., \& BENSON, F. (1986). The frontal lobes. New York: Raven Press.

STUSS, D., EsKES, G., \& FosTER, J. (1994). Experimental neuropsychological studies of frontal lobe functions. In F. Boller \& J. Grafman
(Eds.), Handbook of neuropsychology (Vol. 9, pp. 149-185). Amsterdam: Elsevier.

TEUBER, H.-L. (1964). The riddle of frontal lobe function in man. In J. M. Warren \& K. Akert (Eds.), The frontal granular cortex and behavior (pp. 410-444), New York: McGraw-Hill.

Thompson-Schill, S. L., Swick, D., Farah, M. J., D'Esposito, M., KAN, I. P., \& KNIGHT R. T. (1998). Verb generation in patients with focal frontal lesions: A neuropsychological test of neuroimaging findings. Proceedings of the National Academy of Sciences, 95, $15855-15860$.

Tulving, E., Kapur, S., Craik, F. I. M., Moscovitch, M., \& Houle, S. (1994). Hemispheric encoding/retrieval asymmetry in episodic memory: Positron emission tomography findings. Proceedings of the $\mathrm{Na}$ tional Academy of Sciences, 91, 2016-2020.

UNGERLEIDER, L. G., \& MishKIN, M. (1982). Two cortical visual systems. In D. J. Ingle, M. A. Goodale, \& R. J. W. Mansfield (Eds.), The analysis of visual behavior (pp. 549-586). Cambridge, MA: MIT Press.

Wagner, A. D., Schacter, D. L., Rotte, M., \& Koutstaal, W. (1998). Building memories: Remembering and forgetting of verbal experiences as predicted by brain activity. Science, 281, 1188-1191.

WALKER, A. E. (1940). A cytoarchitectural study of the prefrontal area of the macaque monkey. Journal of Comparative Neurology, 73, 5989.

Wilson, F. A. W., O'Scal.aidhe, S. P., \& Goldman-Rakic, P. S. (1993). Dissociation of object and spatial processing domains in primate prefrontal cortex. Science, 260, 1955-1958.

YAMAGUCHI, S., \& KNIGHT, R. T. (1990). Gating of somatosensory inputs by human prefrontal cortex. Brain Research, 521, 281-288.

(Manuscript received October 14, 1999; revision accepted for publication February 29,2000 .) 